\title{
Effects of Non-uniform Pollution on the AC Flashover Performance of Suspension Insulators
}

\author{
Zhang Zhijin ${ }^{\dagger}$, Zhao Jiayao*, Wei Donghong* and Jiang Xingliang*
}

\begin{abstract}
The non-uniform distribution of contamination on insulator surface has appreciable effects on flashover voltage, and corresponding researches are valuable for the better selection of outdoor insulation. In this paper, two typical types of porcelain and glass insulators which are widely used in ac lines were taken as the research subjects, and their corrections of AC flashover voltage under nonuniform pollution were studied. Besides, their flashover characteristics under different ratio $(T / B)$ of top to bottom surface salt deposit density $(S D D)$ were investigated, including the analysis of flashover voltage, surface pollution layer conductivity and critical leakage current. Test results gave the modified formulas for predicting flashover voltage of the two samples, which can be directly applied in the transmission line design. Also, the analysis delivered that, the basic reason why the flashover voltage increases with the decrease of $T / B$, is due to the decrease of equivalent surface conductivity of the whole surface and the decrease of critical leakage current. This research will be of certain value in providing references for outdoor insulation selection, as well as in proposing more information for revealing pollution flashover mechanism.
\end{abstract}

Keywords: Insulator, Salt deposit density, Non-uniform pollution, Flashover voltage, Surface layer conductivity, Critical leakage current

\section{Introduction}

In recent years, along with the rapid development of industry and economy, the air quality is getting worse, and the accident caused by pollution flashover occurs from time to time in China and around the world. These pollution flashovers may cause large-scale blackouts accident of the grid system [1,2]. Given this, plenty of studies on the pollution flashover performance and mechanism have been done in many countries [3].

The pollution accumulation experiments of field operating insulators delivered that the contamination on insulators in service is always non-uniform. Thus some researches were conducted to discuss the performance of non-uniform contamination on top and bottom surfaces, as well as its influence on insulator flashover [4-16].

For example, according to the dc operation experience in China, researchers in [8] found that, for porcelain and glass insulator, the contamination ratio $(T / B)$ of top to bottom surface of porcelain and glass insulators is generally in range of 1:5 - 1:10; test results in [10] indicated that, under non-uniform pollution on top and bottom surface, the pollution withstand voltage increases by $30 \%$ and $50 \%$ respectively when $T / B$ is 1:5 and 1:10; EPRI [11] raised a

$\dagger \quad$ Corresponding Author: State Key Laboratory of Power Transmission Equipment \& System Security and New Technology, Chongqing University, Chongqing, China. (zhangzhijing@cqu.edu.cn)

* State Key Laboratory of Power Transmission Equipment \& System Security and New Technology, Chongqing University, Chongqing, China.(1436194406@qq.com, weidonghong2014@163.com, xlljiang@cqu.edu.cn)

Received: November 13, 2015; Accepted: February 4, 2016 formula,

$$
\mathrm{K}=\frac{U_{2}}{U_{1}}=1-C \times \log \left(\frac{T}{B}\right)
$$

for the correction of de flashover voltage under nonuniform pollution, and got that the correction coefficient (C) was in the range of 0.29-0.47; in [12], another research found that the formula of EPRI is also applicable in ac case and the value of $\mathrm{C}$ was obtained as 0.31 .

Some works of the non-uniform pollution have been done around the world, and the related data in specific to certain kinds of insulators were referable in outdoor insulation design. However, their results are of some discrepancy. For example, in [10], the value of $\mathrm{C}$ for ceramic insulators ranges from 0.24 to 0.29 and for glass insulators it ranges from 0.18 to 0.20 , while in [12], the correction coefficient $\mathrm{C}$ for ceramic insulators is in the range of $0.21-0.37$. Therefore, more tests were needed to provide detailed information for the selection of correction coefficient.

In previous work, few works have focused on the effect of the surface pollution layer conductivity and the critical leakage current on the flashover characteristic. For example, in [1], the relationship between surface layer conductivity and equivalent salt deposit density on the uniform condition was studied, while in [2], the influence of atmospheric parameters, such as air density humidity and temperature, on the dielectric strength of insulators was presented. Whereas, the data of surface pollution layer 
conductivity as well as the critical leakage current, are necessary for better understand insulator flashover performance under non-uniform pollution. In this paper, the effects of non-uniform pollution on the $\mathrm{AC}$ flashover performance of two typical types of insulators was revealed through analyzing the surface layer conductivity and critical leakage current.

Given this, ac pollution flashover performance of two typical types of porcelain and glass insulators, which are mostly used in the $110 \mathrm{kV}, 220 \mathrm{kV}$ and $500 \mathrm{kV}$ ac transmission lines in China, were studied in this paper, and the influence of non-uniform pollution distribution was systematically analyzed. Modified formulas for the two typical insulators were proposed, which are directly referable for outdoor insulation design. Research results are of certain value in providing references for engineering practice, as well as in proposing more information for revealing pollution flashover mechanism.

\section{Insulator Samples, Experimental Setup and Procedures}

\subsection{Insulator Samples}

The samples were two typical types of suspension insulators. The technical parameters and profiles of the samples are shown in Table 1 and Fig. 1, in which $H$ is the configuration height, $L$ is the leakage distance and $D$ is the diameter of insulators.

\subsection{Experimental setups}

The tests were carried out in the multi-function artificial climate chamber. The artificial climate chamber, with a diameter of $7.8 \mathrm{~m}$ and a height of $11.6 \mathrm{~m}$, can simulate complex atmospheric environments such as fog, rain, ice and high altitude [13-16]. The power was supplied by the AC voltage test set (YDTW) - $500 \mathrm{kV} / 2000 \mathrm{kVA}$ pollution test transformer, of which the maximum short current is 75

Table 1. Technical parameters of the samples

\begin{tabular}{c|c|c|c|c}
\hline Type & Material & $H(\mathrm{~mm})$ & $D(\mathrm{~mm})$ & $L(\mathrm{~mm})$ \\
\hline Type A: $\mathrm{XXY}_{4}-160$ & Glass & 146 & 280 & 450 \\
\hline Type B: XP-160 & Porcelain & 155 & 255 & 305 \\
\hline
\end{tabular}

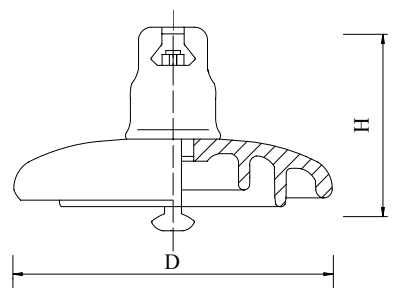

(a)

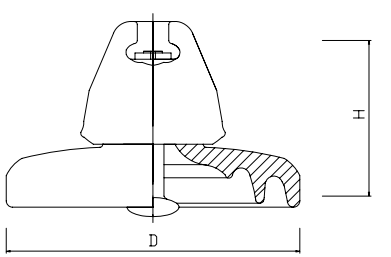

(b)
Fig. 1. Structure of the two samples: (a) Type A; (b) Type B.

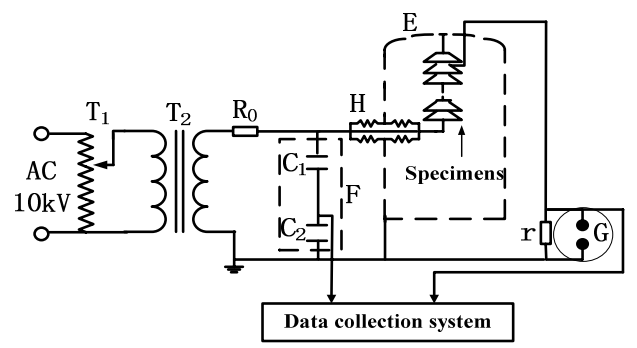

Fig. 2. AC pollution flashover test circuit.

A. The applied voltage on test samples was supplied by a $50 \mathrm{~Hz}$ AC power. The test circuit is shown in Fig. 2. In the test circuit, $T_{1}$ is the $10 \mathrm{kV} / 2000 \mathrm{kVA}$ voltage regulator, $\mathrm{T}_{2}$ is the $500 \mathrm{kV} / 2000 \mathrm{kVA}$ ac testing transformer, $\mathrm{C}_{1}(150 \mathrm{pF})$ and $\mathrm{C}_{2}(1.5 \mu \mathrm{F})$ are the capacitors of the capacitor divider $\mathrm{F}$ $(10000: 1), \mathrm{H}$ is a wall bushing $(330 \mathrm{kV}), \mathrm{R}_{0}(10 \mathrm{k} \Omega)$ is a current limiting resistor, $\mathrm{G}$ is a protective discharge tube (voltage rating $5 \mathrm{~V}), \mathrm{r}(1 \Omega$ ) is a current sampling resistor, $\mathrm{E}$ is the artificial climate chamber. The setups meet the requirements of pollution flashover test [17-18].

\subsection{Test Procedures}

\subsubsection{Preparation}

Before the tests, all the samples were carefully cleaned by $\mathrm{Na}_{2} \mathrm{PO}_{3}$ solution so that all traces of dirt and grease were removed. Then the samples were thoroughly rinsed with tap water, and let to dry naturally indoor to avoid dust or other pollution.

\subsubsection{Pollution}

The soluble contaminants were calculated by soluble deposit density $(S D D)$. SDD represents the weight of soluble materials per unit area of insulator, in ' $\mathrm{mg} / \mathrm{cm}^{2}$, And non-soluble materials were still calculated by nonsoluble deposit density (NSDD). SDD was selected as 0.06 , 0.10 and $0.25 \mathrm{mg} / \mathrm{cm}^{2}$ to represent three different levels of pollution. The ratio of $N S D D$ to $S D D$ was 3.5 in all the tests. The contamination ratio $(T / B)$ of top to bottom surface of porcelain and glass insulators was generally selected for $1: 1,1: 3,1: 5,1: 8$ and $1: 15$ to represent five different degrees of pollution.

The insulators were polluted by solid layer method using brush. The tests used $\mathrm{NaCl}$ to represent soluble contaminant, and Kieselguhr to represent non-soluble contaminant. The mathematic relationship between soluble deposit density of top and bottom surface $\left(S D D_{T} / S D D_{B}\right)$ and the average soluble deposit density $(S D D)$ of the porcelain and glass insulators can be expressed as follows:

$$
\left\{\begin{array}{l}
\mathrm{SDD} \quad=\frac{S D D_{T} \cdot S_{T}+S D D_{B} \cdot S_{B}}{S_{T}+S_{B}} \\
\frac{T}{B}=\frac{S D D_{T}}{S D D_{B}}
\end{array}\right.
$$


where $S_{T}, S_{B}$ are the area of top and bottom surface of the porcelain and glass insulators.

\subsubsection{Wetting}

Natural drying of the samples was ensured to be sufficient. Then the samples were suspended into the climate chamber. The polluted insulators were wetted by steam fog which was generated by a $1.5 \mathrm{t} / \mathrm{h}$ boiler. The fog input rate was $0.05 \pm 0.01 \mathrm{~kg} / \mathrm{h}^{\circ} \mathrm{m}^{3}$, and the temperature in the chamber was controlled between $30^{\circ} \mathrm{C}$ and $35^{\circ} \mathrm{C}$ through the refrigeration system and the atmospheric pressure is $98.6 \mathrm{kPa}$ in all the experiments.

\subsubsection{Flashover test}

The flashover tests were carried out right after the pollution layer was completely wet. In the tests, up and down method was adopted [17-18]. Each contaminated sample was subjected to at least 15 "valid" individual tests. The voltage step was approximately $5 \%$ of the expected $U_{50}$. The first "valid" individual test was selected as being the first one that yields a result different from the preceding ones. Only the individual test and at least 14 following individual tests were taken as useful tests to determine $U_{50}$. The $U_{50}$ and relative standard deviation error $(\sigma)$ are calculated as follows:

$$
\sigma=\frac{U_{50}=\frac{\sum\left(U_{i} n_{i}\right)}{N}}{\sqrt{\left(\sum_{i=1}^{N}\left(U_{i}-U_{50}\right)^{2}\right) /(N-1)}} \times 100 \%
$$

where $U_{i}$ is an applied voltage level, $n_{i}$ is the number of tests carried out at the same applied voltage $U_{i}$, and $N$ is the total number of "valid" tests.

For example, the scatter plot below demonstrate the flashover result of type-B insulator when $T / B$ is $1: 1, S D D$ is $0.1 \mathrm{mg} / \mathrm{cm}^{2}$. As the figure showed that the second test result is different from the first one, so it can be classified as valid results as well as the 18 results after it. Then $U_{50}$

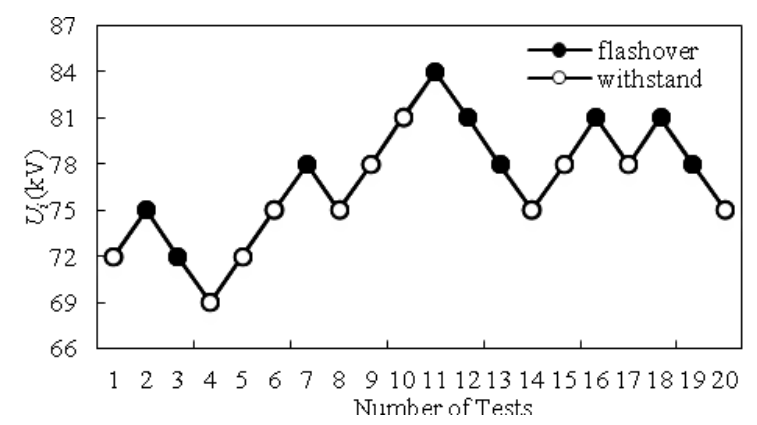

Fig. 3. Example of calculating $U_{50}$ using up and down method and relative standard deviation error $(\sigma)$ can be calculated through those 19 valid results using Eq. (3), (4).

$$
\begin{gathered}
U_{50}=\frac{\sum\left(U_{i} n_{i}\right)}{N}=77.0 \mathrm{kV} \\
\sigma=\frac{\sqrt{\left(\sum_{i=1}^{N}\left(U_{i}-U_{50}\right)^{2}\right) /(N-1)}}{U_{50}} \times 100 \%=4.5 \%
\end{gathered}
$$

\section{Test Results and Analysis}

\subsection{AC Flashover voltage results}

Following the procedures above, ac flashover tests of 7unit insulator strings polluted under different $S D D$ and contamination ratio $(T / B)$ of top to bottom surface were carried out. The results are shown in Table 2 and Table 3.

Table 2. Test results of 7-unit A-type insulator string

\begin{tabular}{c|c|c|c|c|c|c}
\hline \multirow{2}{*}{$\mathrm{T} / \mathrm{B}$} & \multicolumn{2}{|c|}{$\mathrm{SDD}=0.06 \mathrm{mg} / \mathrm{cm}^{2}$} & \multicolumn{2}{c|}{$\mathrm{SDD}=0.1 \mathrm{mg} / \mathrm{cm}^{2}$} & \multicolumn{2}{c}{$\mathrm{SDD}=0.25 \mathrm{mg} / \mathrm{cm}^{2}$} \\
\cline { 2 - 7 } & $\mathrm{U}_{50}(\mathrm{kV})$ & $\sigma(\%)$ & $\mathrm{U}_{50}(\mathrm{kV})$ & $\sigma(\%)$ & $\mathrm{U}_{50}(\mathrm{kV})$ & $\sigma(\%)$ \\
\hline $1: 1$ & 102.5 & 6.5 & 90.8 & 5.3 & 68.8 & 5.6 \\
\hline $1: 3$ & 116.1 & 6.7 & 98.9 & 5.7 & 77.5 & 5.9 \\
\hline $1: 5$ & 121.6 & 7.1 & 105.3 & 6.2 & 81.9 & 6.1 \\
\hline $1: 8$ & 127.2 & 7.6 & 110.3 & 7.5 & 85.9 & 6.9 \\
\hline $1: 15$ & 133.2 & 6.8 & 118.1 & 7.7 & 89.3 & 7.4 \\
\hline
\end{tabular}

Table 3. Test results of 7-unit B-type insulator string

\begin{tabular}{c|c|c|c|c|c|c}
\hline \multirow{2}{*}{$\mathrm{T} / \mathrm{B}$} & \multicolumn{2}{|c|}{$\mathrm{SDD}=0.06 \mathrm{mg} / \mathrm{cm}^{2}$} & \multicolumn{2}{c}{$\mathrm{SDD}=0.1 \mathrm{mg} / \mathrm{cm}^{2}$} & \multicolumn{2}{c}{$\mathrm{SDD}=0.25 \mathrm{mg} / \mathrm{cm}^{2}$} \\
\cline { 2 - 7 } & $\mathrm{U}_{50}(\mathrm{kV})$ & $\sigma(\%)$ & $\mathrm{U}_{50}(\mathrm{kV})$ & & $\mathrm{U}_{50}(\mathrm{kV})$ & $\sigma(\%)$ \\
\hline $1: 1$ & 94.1 & 4.1 & 77.0 & 4.5 & 55.5 & 5.5 \\
\hline $1: 3$ & 116.5 & 4.7 & 93.2 & 5.7 & 69.2 & 5.8 \\
\hline $1: 5$ & 127.8 & 6.7 & 102.5 & 6.8 & 75.0 & 7.3 \\
\hline $1: 8$ & 135.4 & 7.3 & 111.7 & 6.4 & 81.1 & 6.9 \\
\hline $1: 15$ & 150.2 & 7.4 & 120.3 & 7.8 & 88.2 & 7.7 \\
\hline
\end{tabular}

From the test results, conclusions can be made as follows:

(1) The relative standard deviations of these results are all less than $8 \%$, which means that the dispersion of the test results is slight.

(2) The flashover voltage of insulator string decreases with the increase of $S D D$ under a certain value of $\mathrm{T} / \mathrm{B}$. Take B-type insulator string for example, when $T / B=1: 3$, the value of $S D D$ is $0.06,0.10$ and $0.25 \mathrm{mg} / \mathrm{cm}^{2}$, the corresponding $U_{50}$ is $116.5 \mathrm{kV}, 93.2 \mathrm{kV}$ and $69.2 \mathrm{kV}$ respectively, which means that the voltage decreases by $20.0 \%$ and $40.6 \%$ when the $S D D$ increases from 0.06 to 0.10 and $0.25 \mathrm{mg} / \mathrm{cm}^{2}$ correspondingly.

(3) $\mathrm{U}_{50}$ was remarkably affected by the contamination ratio $(T / B)$ of top to bottom surface of insulator, and the lower the $T / B$ ratio, the higher the $U_{50}$ of insulator strings. Take A-type insulator string for example: when $S D D$ is $0.10 \mathrm{mg} / \mathrm{cm}^{2}$, and the $T / B$ ratio changes from 
$1 / 1,1 / 3,1 / 5,1 / 8$ to $1 / 15$ respectively, the $U_{50}$ is $90.8 \mathrm{kV}$, $98.9 \mathrm{kV}, \quad 105.7 \mathrm{kV}, \quad 110.3 \mathrm{kV}$ and $118.1 \mathrm{kV}$ correspondingly. The data shows that when the $T / B$ ratio decreases from $1 / 1$ to $1 / 3,1 / 5,1 / 8$ and $1 / 15$, the $U_{50}$ will increase by $7.7 \%, 15.1 \%, 20.2 \%$ and $28.6 \%$ correspondingly.

\subsection{Relationship between $T / B$ and $S P L C$}

The non-uniformity $T / B$ on the top and bottom surface of insulators affects its pollution flashover voltage, and the relationship between them is shown in Fig. 4.

It can be seen from Fig. 4 that the AC flashover voltage grows with the decreases of $T / B$.

Eq. (2) shows that, when $T / B<1, S D D_{B}>S D D$ and $S D D_{T}<S D D$. The top and bottom surface of the samples were all uniformly coated with $\mathrm{NaCl}$ in the tests, so their surface pollution layer conductivity $(S P L C)$ is directly proportional to $S D D$ when they are at the same temperature and saturated sufficiently [12]. In other words, an increase of $T / B$ causes the layer conductivity of insulator top surface to decrease, and the bottom surface vice versa.

The relationship between the shape factor of insulator $(f)$, the conductivity of pollution layer $(\gamma)$, the surface conductance $(G)$ and the resistance of pollution layer $(R)$ satisfy [19]:

$$
\gamma=f \times G=\frac{1}{R} \times f
$$

The resistance of the whole surface pollution layer consists of the resistance of the top surface in series with that of the bottom surface. Thus from Eq. (5), the equivalent conductivity of the whole insulator surface $\left(\gamma_{\text {eq non }}\right)$ can be obtained:

$$
\gamma_{\text {eq_non }}=\frac{f}{\frac{f_{\mathrm{T}}}{\gamma_{\mathrm{T}}}+\frac{f_{\mathrm{B}}}{\gamma_{\mathrm{B}}}}
$$

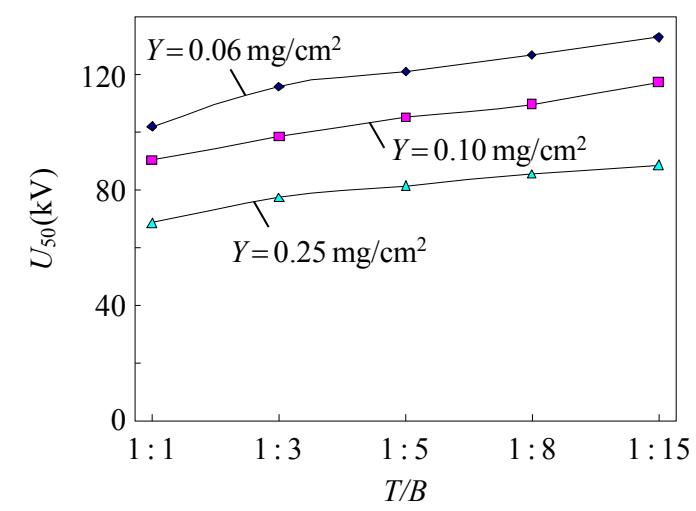

Fig. 4. Relationship between the flashover voltage and $T / B$ for A-type insulator. where $\gamma_{T}$ is the conductivity of top surface and $\gamma_{B}$ is that of bottom surface. The shape factor $(f)$ and the profile of the insulators can be expressed by [20]:

$$
\left\{\begin{array}{l}
f=\int_{0}^{L} \frac{d l}{\pi D(l)} \\
f=f_{\mathrm{T}}+f_{\mathrm{B}}
\end{array}\right.
$$

where $L$ is the insulator surface creepage distance, $\mathrm{dl}$ is the increment of creepage distance, $D(l)$ represents the diameter at distance $d l, f_{T}$ and $f_{B}$ is the shape factor of the top and bottom surface of insulator respectively, $f$ is the total shape factor of insulator.

Since the conductivity of pollution layer $(\gamma)$ is proportional to $S D D$, according to Eq. (2), (5), (7) and (8), the equivalent conductivity ratio $(K)$ of the whole insulator surface under non-uniform pollution distribution to that of uniform pollution can be expressed by the function of $S D D$, $S D D_{T}$ and $S D D_{B}$ :

$$
K=\frac{\gamma_{\text {eq_non }}}{\gamma_{\text {eq_uni }}}=\frac{f \times S D D_{\mathrm{T}} S D D_{\mathrm{B}}}{S D D\left(f_{\mathrm{B}} S D D_{\mathrm{T}}+f_{\mathrm{T}} S D D_{\mathrm{B}}\right)}
$$

Following Standard [20] and the insulator structure in Table 1, the related technical parameters of the samples can be calculated. For A-type insulator, $f, f_{T}$ and $f_{B}$ are 0.748 , 0.203 and 0.545 respectively, while For B-type insulator, they are $0.702,0.210$ and 0.492 correspondingly.

Table 4. Value $K$ of the two samples in different $T / B$.

\begin{tabular}{c|c|c|c|c|c}
\hline \multirow{2}{*}{ Type } & \multicolumn{5}{|c}{$K$} \\
\cline { 2 - 6 } & $T / B=1: 1$ & $T / B=1: 3$ & $T / B=1: 5$ & $T / B=1: 8$ & $T / B=1: 15$ \\
\hline Type A & 1 & 0.859 & 0.680 & 0.509 & 0.318 \\
\hline Type B & 1 & 0.879 & 0.696 & 0.520 & 0.323 \\
\hline
\end{tabular}

With values of $f, f_{T}$ and $f_{B}$, the ratio $K$ can be calculated using Eq. (8) as shown in Table 4. It can be seen that the mean pollution surface conductivity along the whole surface of insulator will get smaller if the non-uniformity of the pollution distribution between the top and bottom surfaces increases. For example, when $T / B$ is $1 / 3,1 / 5,1 / 8$ and $1 / 15$ respectively, the $K$ of A-type insulator is 0.859 , $0.680,0.509$ and 0.318 correspondingly, which means that the comprehensive function of non-uniform pollution is to make the mean conductivity of the whole surface pollution layer decrease with the decrease of $T / B$. Therefore, under the same applied voltage, the leakage current may decrease with the decrease of $T / B$, making it more difficult for dry band to appear on the pollution layer.

\subsection{Relationship between $T / B$ and $I_{C R}$}

Leakage current is an important parameter of electrical property test, which contains the information of insulators operational status. In this paper, the leakage current just 


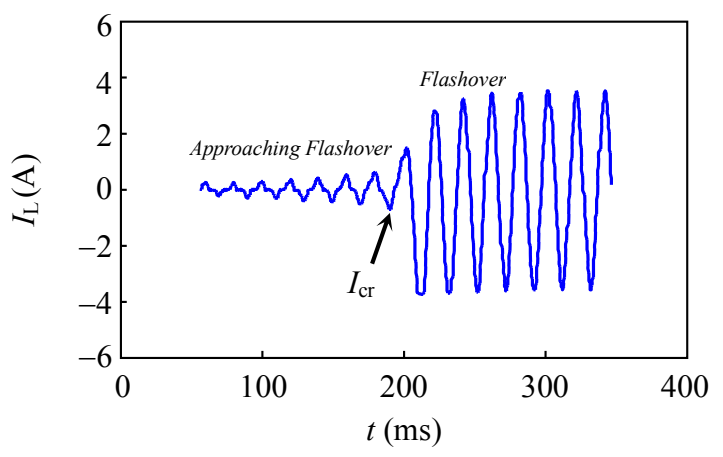

Fig. 5. Waveform of leakage current along the surface of insulator.

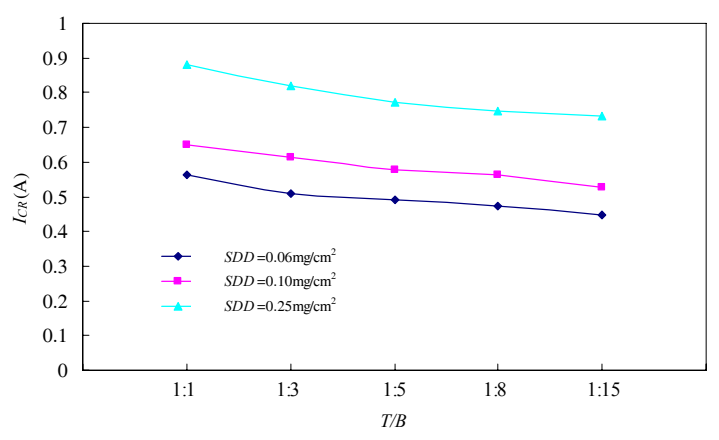

Fig. 6. Relationship between $I_{C R}$ and $T / B$ of for B-type insulator string.

before flashover, namely the critical leakage current $I_{C R}$, was selected as the characteristic parameter of discharge process, and the influence of $T / B$ on $I_{C R}$ was analyzed. Fig. 5 shows the waveform of leakage current during the flashover process when $S D D$ is $0.1 \mathrm{mg} / \mathrm{cm}^{2}, T / B$ is $1: 1$ of type-B insulator. Generally, the peak value at the first half cycle before flashover is defined as critical leakage current $I_{C R}$, as is marked in Fig. 5. During the test, $I_{C R}$ of each flashover test were recorded, and the mean values corresponding to each pollution condition were calculated and shown in Fig. 6.

It can be indicated from Fig. 6 that, under a certain $S D D$, $I_{C R}$ decreases with the decrease of $T / B$. Take A-type insulator for example, when $S D D=0.06 \mathrm{mg} / \mathrm{cm}^{2}, T / B=1: 1$, the critical leakage current values is $0.595 \mathrm{~mA}, 0.526 \mathrm{~mA}$, $0.507 \mathrm{~mA}, 0.489 \mathrm{~mA}$ and $0.478 \mathrm{~mA}$ respectively when $T / B$ decreases from $1 / 1,1 / 3,1 / 5,1 / 8$ to $1 / 15$. The change of $I_{C R}$ is also due to the influence of non-uniform pollution layer on the mean conductivity of the whole surface pollution layer.

From the mathematic flashover model in [21], the basic equation to maintain the $\mathrm{AC}$ arc along the polluted insulator can be expressed as follows:

$$
U_{m}=U_{a r c_{-} m}+U_{p_{-} m}=A x I_{m}^{-n}+r_{a}(L-x) I_{m}
$$

where $U_{m}$ and $I_{m}$ are the peak value of the applied voltage and the leakage current; $U_{\text {arc } m}$ is the voltage on the arc;
$U_{p m}$ is the voltage on the residual pollution resistance. $A$ and $n$ are the arc constant; $L$ is the total creepage distance; $x$ is the length of the arc; $r_{a}$ is the residual pollution resistance per unit length.

Moreover, the partial arc propagation criterion is [22]:

$$
E_{p}>E_{\mathrm{arc}}
$$

where $E_{p}$ is voltage gradient of the residual contaminated parts, $E_{a r c}$ is arc gradient. $E_{p}$ and $E_{\text {arc }}$ can be expressed as [22]:

$$
\begin{aligned}
& E_{p}=\frac{U_{p_{-} m}}{L-x}=\frac{r_{a}(L-x) I_{m}}{L-x}=r_{a} I_{m} \\
& E_{\text {arc }}=\frac{U_{\text {arc } m} m}{x}=\frac{A I_{m}^{-n} x}{x}=A I_{m}{ }^{-n}
\end{aligned}
$$

According to the Eq. (11) and (12), the lower value of leakage current, the harder the Inequality (10) can be satisfied. It makes the partial arc propagation on the surface of polluted insulator difficult. Therefore for the insulator with lower $T / B$, the arc propagation criterion is hard to be satisfied, the applied voltage should be increased to increase the leakage current and satisfy the arc propagation criterion.

\section{Flashover Voltage Correction under Non-uniform Pollution}

The relationship between $U_{50}$ and $S D D$ under different $T / B$ can be indicated from the test data, as is shown in Fig. 7. This figure shows that under a certain $T / B$, insulator strings flashover voltage $U_{50}$ decreases with the increase of $S D D$.

Insulator flashover voltage and salt deposit density meet negative exponent function:

$$
U_{50}=a \cdot S D D^{-b}
$$

where a is a coefficient associated with insulator profile and environment conditions, $\mathrm{b}$ is characteristic exponent characterizing the influence of $S D D$ on $U_{50}$.

Therefore, through fitting the curves in Fig. 7 by Eq. (13), the coefficient a, the influence characteristic exponent

Table 5. Results of $a$ and $b$ of A-type insulator string in different $T / B$

\begin{tabular}{c|c|c|c}
\hline $\mathrm{T} / \mathrm{B}$ & $a$ & $b$ & $R^{2}$ \\
\hline $1: 1$ & 46.76 & 0.282 & 0.9964 \\
\hline $1: 3$ & 52.29 & 0.281 & 0.9981 \\
\hline $1: 5$ & 55.78 & 0.277 & 0.9998 \\
\hline $1: 8$ & 58.66 & 0.275 & 0.9999 \\
\hline $1: 15$ & 60.63 & 0.283 & 0.9960 \\
\hline
\end{tabular}




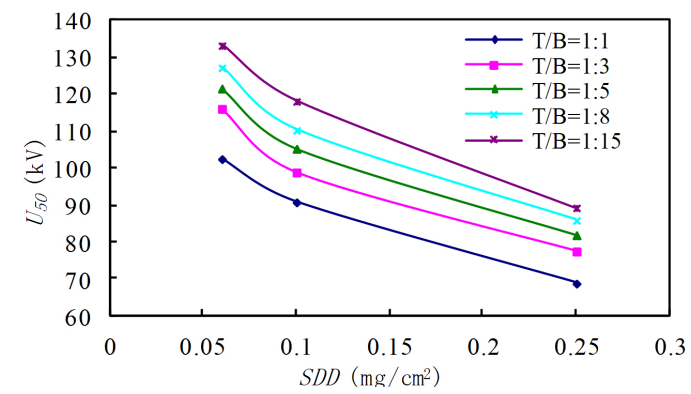

(a)

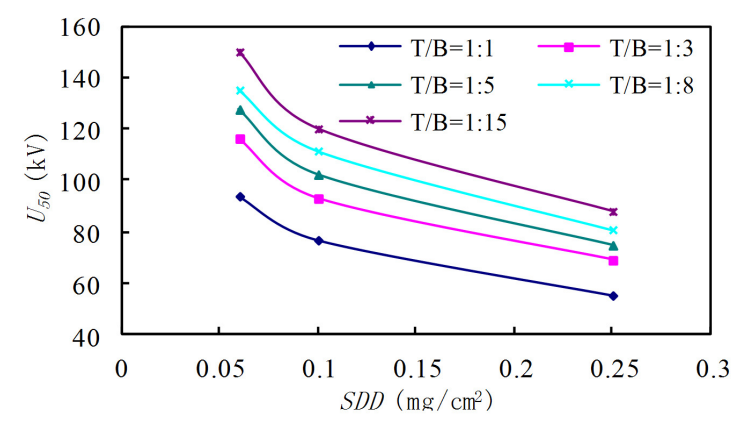

(b)

Fig. 7. Relationship between $U_{50}$ and $S D D$ : (a) A-type insulator; (b) B-type insulator.

Table 6. Values of $a$ and $b$ of B-type insulator string in different $T / B$

\begin{tabular}{c|c|c|c}
\hline T/B & $a$ & $b$ & $R^{2}$ \\
\hline $1: 1$ & 33.21 & 0.369 & 0.9994 \\
\hline $1: 3$ & 41.64 & 0.360 & 0.9936 \\
\hline $1: 5$ & 44.74 & 0.369 & 0.9958 \\
\hline $1: 8$ & 49.27 & 0.358 & 0.9996 \\
\hline $1: 15$ & 52.50 & 0.369 & 0.9955 \\
\hline
\end{tabular}

$\mathrm{b}$ and the fitting degree $\mathrm{R}^{2}$ of each sample in each $T / B$ condition can be obtained, as shown in Table 5 and Table 6 .

The fitting results reveal that the influence of $T / B$ and $S D D$ on ac flashover voltage should be independent. Take B-type insulator for example, the $b$ values are $0.369,0.360$, $0.369,0.358$ and 0.369 respectively when $T / B$ are $1 / 1,1 / 3$, $1 / 5,1 / 8$ to $1 / 15$ correspondingly, and the mean value of $b$ is 0.365 . The relative errors between the $b$ values and its mean value are just within $1.10 \%$ and $-1.92 \%$, which are very small, so the influence of $T / B$ on $b$ is not obvious. In other words, $b$ can be treated as a constant while $T / B$ is changing. Therefore, the functions of $T / B$ and $S D D$ on $U_{50}$ can be seen to be independent. According to Eq. (1) and (13), the calculation of $U_{50}$ under non-uniform pollution can be expressed as follows:

$$
U_{50}=a \cdot S D D^{-b}[1-C \cdot \log (T / B)]
$$

Some mathematical methods and the fitting analysis based on Eq. (14) were adopted for the test data in Table 2
Table 7. Calculating errors between the test values and the calculated values

\begin{tabular}{c|c|c|c|c|c|c}
\hline \multirow{2}{*}{ Type } & $S D D$ & \multicolumn{5}{|c}{$\Delta U(\%)$} \\
\cline { 2 - 7 } & $\left(\mathrm{mg} / \mathrm{cm}^{2}\right)$ & $T / B=1: 1$ & $T / B=1: 3$ & $T / B=1: 5$ & $T / B=1: 8$ & $T / B=1: 15$ \\
\hline \multirow{2}{*}{ Type } & 0.25 & -0.67 & -0.09 & -0.59 & -0.36 & -2.27 \\
\cline { 2 - 7 } A & 0.10 & 1.41 & -2.21 & -1.12 & -1.02 & 0.04 \\
\cline { 2 - 7 } & 0.06 & -0.76 & -0.46 & -1.02 & -1.07 & -2.25 \\
\hline \multirow{2}{*}{$\begin{array}{c}\text { Type } \\
\text { B }\end{array}$} & 0.25 & 0.06 & 0.62 & 0.21 & 0.60 & -0.04 \\
\cline { 2 - 7 } & 0.10 & -0.70 & -3.09 & -2.14 & -0.82 & -2.44 \\
\cline { 2 - 7 } & 0.06 & 0.74 & 0.63 & 1.29 & -0.19 & 1.15 \\
\hline
\end{tabular}

and 3 , and then the equations for predicting the $U_{50}$ of Atype and B-type insulators can be got:

$$
\begin{array}{ll}
U_{50}=46.98 \cdot S D D^{-0.280}[1-0.271 \cdot \log (T / B)] & \text { TуреA } \\
U_{50}=33.46 \cdot S D D^{-0.365}[1-0.501 \cdot \log (T / B)] & \text { TуреB }
\end{array}
$$

Define the calculating error as:

$$
\Delta U(\%)=\frac{U_{f T}-U_{f C}}{U_{f T}} \times 100 \%
$$

where $U_{f T}$ is the test value of $U_{50}$ while $U_{f C}$ is its calculated value using Eq. (15) and (16). $\Delta U(\%)$ corresponding to each pollution condition can be calculated, as shown in Table 7 .

It can be inferred from the table that by using Eq. (15) and (16) to calculate the $U_{50}$, the relative error are all within $\pm 3 \%$, which suggests that the two equations for predicting flashover voltage under different $T / B$ and $S D D$ values are acceptable.

\section{Conclusion}

In this paper, the flashover performance of typical type insulators under non-uniform pollution was studied. Through analysis the following conclusions can be obtained:

(1) Both salt deposit density $S D D$ and pollution nonuniformity $T / B$ of insulator have obvious effects on flashover voltage, and their effects are independent from each other.

(2) The relationship among the ac pollution flashover voltage $\left(U_{50}\right), S D D$ and $T / B$ of insulator string meets:

$$
U_{50}=a \cdot S D D^{-b}[1-C \cdot \log (T / B)]
$$

For the two typical types of porcelain and glass insulators, the prediction of $U_{50}$ can be made by:

$$
\begin{array}{ll}
U_{50}=46.98 \cdot S D D^{-0.280}[1-0.271 \cdot \log (T / B)] & \text { Tyре } A \\
U_{50}=33.46 \cdot S D D^{-0.365}[1-0.501 \cdot \log (T / B)] & \text { TyреB }
\end{array}
$$


(3) The non-uniformly distribution of pollution layer on top and bottom surfaces of insulator string causes the decrease of equivalent conductivity of the whole surface. The more uneven the pollution distribution on the top and bottom surface of insulators, the smaller the mean pollution surface conductivity along the whole surface of insulators. In that case, the leakage current is lowered, which restricts the propagation of partial arc and finally causes the flashover voltage to rise.

\section{Acknowledgements}

The authors gratefully acknowledge the contributions of group members for their work on the experiments. The authors also thank the support of the Funds for Innovative Research Groups of China (51321063) and State Grid Corporation of China.

\section{References}

[1] Leclerc M, Bouchard RP, Gervais Y, Mukhedkar D. Wetting processes on a contaminated insulator surface. IEEE Trans. Power Appl Syst 1982; 101: 1005-1011.

[2] Zhang R, Zheng J. Progress in outdoor insulation research in China. IEEE Trans Electr Insul 1990; 25: 1125-1137.

[3] Zhang Z, Jiang X, Cao Y, Sun C, Hu J. Influence of low atmospheric pressure on $\mathrm{AC}$ pollution flashover performance of various types insulators. IEEE Trans Dielectr Electr Insul 2010; 17: 425-433.

[4] Naito K, Hasegawa Y, Imakoma T. Improvement of the DC voltage insulation efficiency of suspension insulators under contaminated conditions. IEEE Trans Electr Insul 1988; 23: 1025-1032.

[5] Douar MA, Mekhaldi A, Bouzidi MC. Flashover process and frequency analysis of the leakage current on insulator model under non-uniform pollution conditions. IEEE Trans Dielectr Electr Insul 2010; 17: 1284-1297.

[6] Chakravorti S, Mukherjee PK. Power frequency and impulse field calculation around a HV insulator with uniform or non-uniform surface pollution. IEEE Trans Electr Insul 1993; 28: 43-53.

[7] Koshairy MAE, Rizk FAM. Comportement des Isolateurs des Lignes de Transport de Très Haute Tension Dans les Conditions Désertiques. CIGRE Rapport 33-05, Paris, France, 1970.

[8] Jiang X, Wang S, Zhang Z, Hu J, Hu Q. Investigation of flashover voltage and non-uniform pollution correction coefficient of short samples of composite insulator intended for $\pm 800 \mathrm{kV}$ UHVDC. IEEE Trans Dielectr Electr Insul 2010; 17: 71-80.
[9] Ye H, Zhang J, Ji Y, Sun W, Kondo K, Imakoma T. Contamination accumulation and withstand voltage characteristics of various types of insulators. In: IEEE 2003 Properties and Applications of Dielectric Materials (ICPADM) Conference, Nagoya, Japan: IEEE. pp. 1019-1023.

[10] Zhang Z, Liu X, Jiang X, Hu J, Gao DW. Study on AC flashover performance for different types of porcelain and glass insulators with non-uniform pollution. IEEE Trans Power Delivery 2013; 28: 1691-1698.

[11] EPRI. HVDC transmission line insulation performance: Report EL24618. EPRI, USA, 1986.

[12] Sima W, Yuan T, Yang Q, Xu K, Sun C. Effect of nonuniform pollution on the withstand characteristics of extra high voltage (EHV) suspension ceramic insulator string. IET Generation, Transmission \& Distribution 2010; 4: 445-455.

[13] Zhang Z, Jiang X, Chao Y, Chen L, Sun C, Hu J. Study on DC pollution flashover performance of various types of long string insulators under low atmospheric pressure conditions. IEEE Trans Power Delivery 2010; 25: 2132-2142.

[14] Liu X. Study on the pollution accumulation rule and AC pollution flashover performance of insulators with typical sheds. MSc, Chongqing University, Chongqing, China, 2013.

[15] Jiang X, Yuan J, Zhang Z, Hu J, Shu L. Study on pollution flashover performance of short samples of composite insulators intended for $\pm 800 \mathrm{kV}$ UHVDC. IEEE Trans Dielectr Electr Insul 2007; 14: 1192-1200.

[16] Jiang X, Yuan J, Shu L, Zhang Z, Hu J, Mao F. Comparison of DC pollution flashover performances of various types of porcelain, glass, and composite insulators. IEEE Trans Power Delivery 2008; 23: 1183-1900.

[17] IEC Standard. Artificial pollution tests on highvoltage insulators to be used on dc Systems. IEC Tech Rep 1245, 1993.

[18] CIGRE WG'S-33.04. Artificial pollution testing of HVDC insulators: analysis of factors influencing performance. Electra 1992; 11: 98-113.

[19] IEC Standard. Artificial pollution tests on highvoltage insulators to be used on AC systems. IEC60507, 2004.

[20] Chinese Standard. Artificial pollution tests on HighVoltage insulators to be used on ac systems solidlayer method, GB/T 4585.2-91, 1991.

[21] Rizk FAM. Mathematical model for pollution flashover. Electra 1981; 78: 101-116.

[22] Rahal AH, Huraux C. Flashover mechanism of HV insulators. IEEE Trans Power App Syst 1979; 98: 2223-2229. 


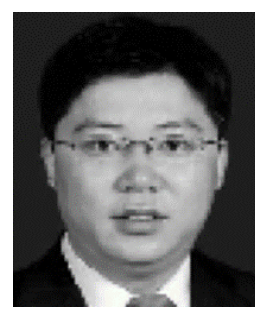

Zhijin Zhang was born in Fujian Province, China, in 1976. He is a member of IEEE. He received the B.Sc., M.Sc., and Ph.D. degrees from Chongqing University, Chongqing, China, in 1999, 2002, and 2007 respectively. Currently, he is a Professor of the College of Electrical Engineering at Chongqing University. His main research interests include high voltage, external insulation, power grid icing. He is the author or coauthor of over 120 papers.

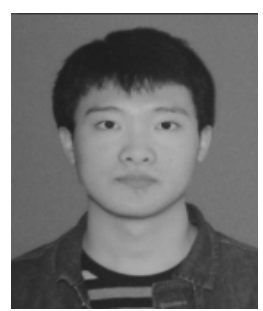

Jiayao Zhao was born in Hebei province, China. He recieved the B.Sc degree from Chongqiong University in 2014. He is currently pursuing the M.Sc - Ph.D degree at Chongqing University. His main research interests include high voltage, external insulation, numerical modeling and simulation.

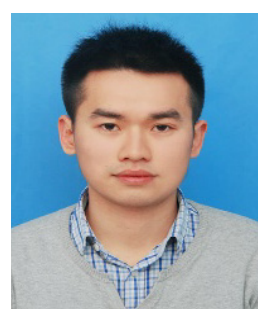

Donghong Wei was born in Chongqing province, China. He received the B.Sc. degree from Chongqiong University in 2014. He is currently pursuing the M.Sc. degree at Chongqing University. His main research interests also include high voltage, external insulation, numerical model-

ing and simulation.

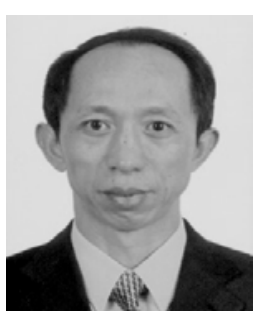

Xingliang Jiang was born in Hunan province, China, on 31 July 1961 . He is a member of IEEE. He graduated from Hunan University in 1982 and got his M.Sc. and Ph.D. degrees from Chongqing University in 1988 and 1997, respectively. His is a professor of College of Electrical Engineering, Chongqing University, Chongqing, China. His special fields of interest include high voltage external insulation, transmission line icing and protection. He has published over 140 papers on his professional work. 